\title{
Kuhn 算法的成本和夏杂性理论
}

我们证明了以下定理。

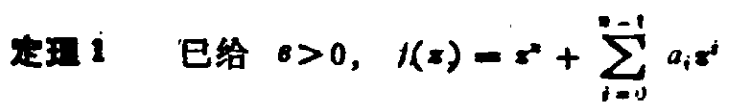
是攵多项式。应用 Kubn 算法,不大于

$$
\begin{aligned}
& {[8 \mid 3(1+\sqrt{2}) n / 4 \pi](1+[3(1+\sqrt{2}) n / 4 \pi])} \\
& +5 \pi \cdot \min \left\{M, \max \left|a_{n}\right|+1+\sqrt{2}\right. \\
& \left.\left.\quad \times\left(1+\frac{3 n}{\pi}\right)\right\}^{2}+28 n d \pi\left(1+\frac{3 n}{2 \pi}\right)^{2}\right]
\end{aligned}
$$

步, $t$ 的某些点可以找到它的 8 通近, 其中

$$
\begin{aligned}
d= & {\left[\log _{2} \sqrt{2}\left(1+\frac{3 n}{2 \pi}\right) / 8\right\}, } \\
M= & \max \{3 \sqrt{2}(2+\pi) n / 4 \pi, \\
& \left.\frac{n}{n-1} \max \left|a_{4}\right|+1\right\}+\sqrt{2}
\end{aligned}
$$

和 $\boldsymbol{r}\rceil$ 表示不小于 $\boldsymbol{r}$ 的船小整数。

定义 1 设 $x_{0} \in C, f(x)=x^{2}+\sum_{i=0}^{n-1} a_{1} x^{i}$ 为 夏多项式, 并归纳定义 $x_{k}=x_{k-1}-f\left(x_{k-1}\right) / f^{\prime}\left(x_{k}\right)$. 如果对所有 $k \geq 1$, 序列 $x_{k}$ 是定义好了的, 并且 当 $k \rightarrow \infty$ 时, $x_{k}$ 收敛到 $f$ 的察点 ** 以及 $\left|f\left(x_{*}\right)\right|$ $f\left(x_{k-1}\right) \mid<1 / 2$, 则称 $x_{0}$ 为 $t$ 的一个通近零点.

尘五2 2 记 $P_{n}(R)=\left\{g(s)=z^{*}+\sum_{i=1}^{n-1} b_{1} s^{i} \mid\right.$ $\left.\left|b_{i}\right|<R, i=0,1, \cdots, n-1\right\}$. 设 $0<\mu<1$ 和 $n$ 为已给, $\sigma=(\mu / n)^{1 / 2}$, 则对任何 $t \in P_{n}(R)$, 应用 Kuhn 算法, 至少以 $1-\mu$ 的概率用至多 s 步可以找 到 $f$ 的 $n$ 个通近

$$
\begin{aligned}
S= & {[8[3(1+\sqrt{2}) n / 4 \pi](1} \\
& +[3(1+\sqrt{2}) n / 4 \pi])+5 \pi \cdot \min \\
& \times\left\{\tilde{M}, R+1+\sqrt{2}\left(1+\frac{3 n}{\pi}\right)\right\}^{\prime} \\
& \left.+28 n d \pi\left(1+\frac{3 \pi}{2 \pi}\right)^{2}\right\}
\end{aligned}
$$

$$
\begin{aligned}
\tilde{M}= & \max \left\{3 \sqrt{2}(2+x) n / 4 \pi, \frac{n}{n-1} R+1\right\} \\
& \quad+\sqrt{2} \\
d= & {\left[\log _{2} \sqrt{2}\left(1+\frac{3 n}{2 \pi}\right) / 8\right], } \\
= & J(1-N)^{2} / 13\left[1-(n+1) N^{n}+n N^{n+1}\right] \\
= & \mu^{\prime 2}(1-N)^{\prime} / 13 n^{1 / 2}\left[1-(n+1) N^{n}\right. \\
\quad & \left.\quad+n N^{n+1}\right], \\
N= & \min \left\{n, R+1+\sqrt{2}\left(1+\frac{3 n}{x}\right)\right\} .
\end{aligned}
$$

定理 3 给定 $0<\mu<1$ 和正整数 $n$ 。假设

$$
\begin{aligned}
& f(s)-\prod_{i=1}^{\pi}\left(s-\xi_{i}\right) \in P_{s}(R) \text {, } \\
& F(R, n)=\frac{1}{R}\left(3 2 ( n + 2 ) \left(R(n-1)(n-2)^{2}\right.\right. \\
& +R+1)^{\prime}+(n-1) \\
& \left.\times(2 n(2+(n+1) R))^{2}\right]^{1 / 2} \text {, } \\
& \rho=\mu^{1 / 2} / F(R, \pi) \text {, } \\
& D=\mid \log _{1}(2 \sqrt{2}) /\left\{\left[(2 R+2)^{n-1}+\rho^{2-1} \mid\right.\right. \\
& \left.13]^{1 /(n 1)}-(2 R+2)\right\} 1 \text {, } \\
& K=|8| 3(1+\sqrt{2}) n / 4 \pi \mid \\
& x(1+|3(1+\sqrt{2}) n / 4 \pi|) \\
& +5 \pi \cdot \min \{\tilde{M}, R+1+\sqrt{2} \\
& \left.\left.\times\left(1+\frac{3 n}{x}\right)\right\}^{2}+28 n D x\left(1+\frac{3 n}{2 \pi}\right)^{2}\right] \text {. } \\
& L=\left(\frac{1}{2}\right)^{D-1 / 2}\left(1+\frac{3 \pi}{2 \pi}\right) \text {, }
\end{aligned}
$$

则以失败的标率不大于 $\mu$, 存在正整数 $K_{1}, \cdots, K_{a}$, $\sum_{i=1}^{n} K_{i} \leqslant K$, 使得如果 $k \geqslant K_{i}, x_{i k}$ 是单调的, 且
(1) $\left|\varepsilon_{i 4}-\xi_{i}\right|<\left(\frac{1}{2}\right)^{\frac{1-K_{i}}{5}} L_{j}$
(2) $\left|t\left(s_{i n}\right)\right|<\left(\frac{1}{2}\right)^{\frac{4-K_{i}}{4}} L(2 R+L+2)^{n-1}$.

徐森林

（中国科技大学数学系, 合肥） 